\title{
GAIA Preterm Labor Levels of Diagnostic Certainty
}

National Cancer Institute

\section{Source}

National Cancer Institute. GAIA Preterm Labor Levels of Diagnostic Certainty. NCI

Thesaurus. Code C127941.

A classification of maternal and fetal outcomes relating to the assessment of preterm labor, developed by the Global Alignment of Immunization safety Assessment in pregnancy, based on the extent to which the diagnosis has been confirmed. 